Proceedings

\title{
Information Ontology as Anti-Metaphysics ${ }^{\dagger}$
}

\author{
Ping Wang * and Jian Wang \\ Department of Philosophy, Xi'an Jiaotong University, Xi'an 710049, China; wangjian0501@163.com \\ * Correspondence: wp56012@stu.xjtu.edu.cn; Tel.: +86-185-9192-0805 \\ + The Fourth International Conference on Philosophy of Information, Berkeley, CA, USA, 2-6 June 2019. \\ Published: 14 May 2020
}

\begin{abstract}
Followed by the general review of various kinds of information ontology as metaphysical thoughts, two main approaches can be illustrated. The basic feature of this kind of metaphysics is its theoretical explanation for the constructivism of the whole world. However, metaphysics is undermining its study of existence itself by taking existence as substantial entities, attempting to construct the whole world, or separating existence and thoughts. With the insight from antimetaphysical approaches, this paper discusses three aspects of the information ontology.
\end{abstract}

Keywords: anti-metaphysics; information; ontology

\section{Current Information Ontologies and Their Metaphysical Generalization}

Every philosophical thought has its ontological requirements. The current international information ontological research is complicated and comprehensive. In summary, it mainly includes the following genres: the first is Wu Kun's information ontology based on materialism, and the second is Dodig-Crnkovic's so called pan-informatics based on info-computationalism, the third is Floridi's information metaphysics related to information epistemology, and the fourth is Hofkirchner, Brier and Deacon's information local ontology related to interdisciplinary research. In addition, such research draws on material-information dualism based on cognitive research, formal ontology based on information science, and information technological ontology on the studies of digital objects.

In brief, the basic points of the Wu's information ontology are: (a) material firstness, information secondness, and the two are unified; (b) the existential domain is divided into three kinds of information, information in itself, information for itself, and regenerated information, where social information is the unification of the three; (c) the discovery of the information world reveals the dual existence and dual evolution of the natural world; (d) in short, any object is both physical and informational, and is dual unity with indirect existence and direct existence [1].

Gordana's info-computationalism holds that: (a) information and computation actually characterize two aspects of one item: information is biased toward abstraction, while computation is biased towards concrete; (b) the universe is composed of information as a continuous stream, converse and communicate mutually by computation; (c) therefore, the universe is defined as a network of computational processing guided by information, which is the product of infinitely multidimensional computational processing.

The basic points of Floridi's information metaphysics include: (a) based on the philosophical basis of Kant's epistemology, semantic information is defined as well-formed and meaningful data; (b) infosphere consisting of information rather than data, forming our basic picture of the worldview; (c) the world itself is unknowable, and human beings are inforgs as agents that coexist in the infosphere with other inforgs.

The main points of the information local ontology of interdisciplinary research are: (a) the belief that information exists only in the field of social interaction, as advocated by brier's cybersemiotics; 
(b) the belief that information does not exist in mechanical physical world but is related to the development of life and its evolution, for example, according to the writings of Terrence Deacon; (c) the belief that information is closely related to the self-organizing system, as long as there is selforganization, there is information-there is no information without self-organization.

In addition, there are many trends similar to dualism. David Chalmers inherits Russell's theory. (a) Dividing information into physical information and information as phenomenon, which are close to each other but not identical; (b) Physical information is not an assertion of the existence of information in the physical world, it is only information in the abstract space of causal interpretation, information as phenomenon is realized in experience, has un-reductive properties; (c) Physical information and information as phenomenon has formal isomorphism, and the realization of information as phenomenon is asymmetrically dependent on physical information. For reasons of space, other points are not repeated here.

\section{Basic Characteristics of Information Metaphysics}

We believe that current information ontology ideas have more or less metaphysical tendencies or meanings, and more or less intrinsic incompleteness. Our views and reasons are:

(a) Dodig-Crnkovic has a general info-computationalism tendency, and this is in line with John Wheeler's "it from bit" and Stony's information cosmology;

(b) Floridi's constructivist approach, while acknowledging the existence of the thing in itself, the world itself, its agnostic and metaphysical features of Kantianism only obscure the ontological problem rather than the solution;

(c) Two-dimensional semantics such as Chalmers and its dualistic thoughts are related to scientism or naturalism, which inevitably have metaphysical construction needs. Barry Smith's formal ontology follows the local ontology of Husserl's philosophy. To some extent, the arrangement of nature is suspended;

(d) The information ontology of Wu Kun directly emphasizes its insistence on the materialist position, but as Engels said, the division of materialism and idealism is meaningful only when it comes to the question of who is first. The proper philosophical position should be considered after the reflection of the meta-ontological problems. At the same time, due to the naturalistic tendency of the philosophy of information, it is easy to cause a metaphysical misunderstanding [2];

(e) In addition, the information local ontology of interdisciplinary research has an ontological incompleteness on the position of information in the world, or a metaphysical meaning of perspectivism [3].

In summary, information metaphysics has the following three difficulties or characteristics:

(a) Because of the problem of Being, that is, the meta-ontological problem has not been considered, an appropriate information ontology has not been established;

(b) A lack of thinking about the ontology of information, laying the foundation for information philosophy with the ontological presupposition under the framework of scientism or idealism;

(c) Either canceling or deviating from the ontology of the world to construct a so-called worldview based on an objective or subjective interpretation of the information.

\section{How Should We Think Anti-Metaphysically?}

In response to the above three questions, our thinking is:

(a) We all recognize that Being is Becoming, existence is generation or process, not entity, the socalled world, that is, the reality of Being, is not an existent field with fixed boundaries, in fact, our world, so it is called the universe, and its time and space are constantly being generated, in an infinite process. We cannot seem to talk about the universe in terms of ontology. It is "all of things or beings" in the universe, the so-called existence, the ontology, but when we think or say "infinite generation" or absolute "nothing", "Generation" and "Nothing" become "existence" 
and "beings". This is the greatest secret of metaphysics, the basic relationship between thought and existence: we cannot talk about existence without thought, in other words, thought is existence.

(b) Thus, as the reality of Being, the world itself is always understood as a world related to thought. The so-called pure external world is a conceptual presupposition of the binary separation of thought and Being. However, this does not mean that the thought gives out the world, or thought comes from nowhere like God's view, as idealists said. And this does not mean that the existence exists as it is without thought, as dogmatist and scientists said. The ontology under those frameworks presuppose the binary separation of thought and existence, made a mistake of delimiting the thoughts or materializing them. In fact, thought is not external of a realization world as existence; in other words, existence is thought.

(c) An appropriate ontology should fully consider the identical relationship between existence and thought. Any way of summarizing the worldview with a certain concept must be metaphysical. The ontological construction related to information research does not mean generalizing information concepts from ontology or from epistemology. We believe that the re-explanation of information ontology should first consider the relationship between information and existence and thought.

\section{The Anti-Metaphysical Explanation of Information Ontology and Its Significance}

Floridi believes that inforgs as agents are onliving in the infosphere, which is composed of information. Like Chalmers, we believe that this infosphere is not a phenomenon of the world's ontology - the Thinking and Being are separated. Therefore, there must be a so-called metaphysical supplement. In contrast, Gordana's thought, while recognizing the identity of the relationship between Thinking and Being, falls into the paradox of idealism. Although it does not explicitly sublate materialism, Wu Kun's information ontology, especially its discussion about the relationship between information and existence and thought, fully respects the research results of natural science and opens up a possible path of dichotomy, and incompleteness errors such as information local ontology are avoided [4].

The anti-metaphysical explanation of information ontology can be carried out in the following aspects:

(a) According to the information epistemology of Wu Kun, information is the intermediary of material and consciousness, the knowing of objects is obtained through information as an intermediary, and is processed and transformed by human cognition [5]. In this sense, it is considered as subjective information. We can see that thoughts are carried out in the form of information. The ability of thought can be seen as a multi-dimensional and multi-level information capability including acquisition, processing, storage, etc., where the thought lies, where the information lies, and the thought is information.

However, this does not mean that all information is consciousness; consciousness is only part of the information, in other words, there is beyond the consciousness.

(b) In the information ontology of Wu Kun, matter is represented in the form of information, and, in Wu Kun's terminology, it can be expressed as follows: information as "indirect existence" is the existent mode and state of matter as "direct existence" represented by itself [6]. At the same time, the material things are also the informational things. The two are the unified relationship of dual existence and dual evolution. That is to say, the information as indirect existence and the matter as direct existence are a dialectical developing relationship that is two-sided rather than directly identical. This does not mean that there is material existence in addition to information, the two together constitute an existent field, but to see the material and information as the two aspects of existence in its process of becoming, that is, the transformation of matter and information condensation or construction in time and space. Here, the becoming or generation of existence is exactly the generation of information. And since the simple and pure existence 
cannot be generated, according to what we call the Being is Becoming, pure existence cannot exist, and thus there is no existence without information.

However, the absence of information means that there is no existence, it does not mean that existence fully equals information, otherwise it will cancel the direct existence and fall into the paninformatics. Here, it only shows that we have no knowledge of existence without information, that is, we have always been indirect to the thought of direct existence, or that, were it not for information, there would be no representation and generation of existence. It is only in this sense that we can say that existence is information.

(c) Combining the information ontology and epistemology of Wu Kun, we can see that, first of all, thought is information, but there is existence beyond consciousness, existence outside the human thought, which is what he calls objective information. Second, existence is information. However, because information, especially objective information, is a self-representation of direct existence, there is no such thing as the simple identical relationship between information and thought and existence. However, we believe that this does not contradict the aforementioned identical principle of Thinking and Being. This is the focus of our explanation of the information ontology, namely:

We believe that the objective information field, which is outside the material and consciousness in the field of traditional ontology, defined by the so-called information world, constitutes the most important breakthrough in information ontology. This discovery makes the philosophy of information beyond the awkwardness of anthropocentrism, and describes the territory that was previously summarized by the concept of "nothing" in human thought with a more specific concept, namely "objective information", to make it into "existence", "beings", "generation", thus becoming the content of a broader concept of "thought".

If we combine its later important ideas about natural value theory and recent developments in intelligent society, we have reason to believe that the theory of the information world laid the foundation for a preliminary ontology of the breakthrough of human consciousness research, or possible artificial intelligence, virtual reality and post-human thoughts.

Conflicts of Interest: The authors declare no conflict of interest.

\section{References}

1. Brenner, J.E. Wu Kun and the metaphilosophy of information. Int. J. Inf. Theor. Appl. 2011, 2, 103-128.

2. Li, W.-G. Information philosophy in China: Professor Wu Kun's 30 years of academic thinking in information philosophy. TripleC 2011, 9, 316-321.

3. Burgin, M. Theory of Information: Fundamentality, Diversity and Unification; World Scientific: Singapore, 2010.

4. $\mathrm{Wu}, \mathrm{K}$. The form of the philosophy of information. Potential Sci. J. 1984, 3, 33-35.

5. $\mathrm{Wu}, \mathrm{K}$. The status and role of information in philosophy. Potential Sci. J. 1981, 3, 53-60.

6. $\mathrm{Wu}, \mathrm{K}$. Formal proposal for a unified complex information theory. Stud. Dialectics Nat. 2006, 12, 96-99.

C 2020 by the authors. Licensee MDPI, Basel, Switzerland. This article is an open access article distributed under the terms and conditions of the Creative Commons Attribution (CC BY) license (http://creativecommons.org/licenses/by/4.0/). 